\title{
Molecular Cloning and Immunogenicity Evaluation of PpiC, GelE, and VS87_01105 Proteins of Enterococcus faecalis as Vaccine Candidates
}

\author{
Hamid Kazemian ${ }^{1}$, Mohammad Reza Pourmand ${ }^{1 *}$, Seyed Davar Siadat ${ }^{2}$, Mehdi \\ Mahdavi ${ }^{3}$, Mohammad Hossein Yazdi ${ }^{4}$, Peyman Avakh Majelan ${ }^{1}$, Davoud Afshar ${ }^{5}$, \\ Mehdi Yaseri $^{6}$, Mehdi Davari ${ }^{2}$ and Muhammad Ibrahim Getso ${ }^{7}$
}

\footnotetext{
${ }^{1}$ Department of Pathobiology, School of Public Health, Tehran University of Medical Sciences, Tehran, Iran;

${ }^{2}$ Department of Mycobacteriology and Pulmonary Research, Pasteur Institute of Iran, Tehran, Iran;

${ }^{3}$ Recombinant Vaccine Research Center, Tehran University of Medical Sciences, Tehran, Iran; ${ }^{4}$ Department of Pharmaceutical Biotechnology and Biotechnology Research Center, Faculty of Pharmacy, Tehran University of Medical Sciences, Tehran, Iran; ${ }^{5}$ Department of Microbiology and Virology, School of Medicine, Zanjan University of Medical Sciences, Zanjan, Iran; ${ }^{6}$ Department of Epidemiology and Biostatistics, School of Public Health, Tehran University of Medical Sciences, Tehran, Iran; ${ }^{7}$ Department of Medical Mycology, School of Public Health, Tehran University of Medical Sciences, International College, Tehran, Iran
}

Received 11 August 2018; revised 16 October 2018; accepted 17 October 2018

\begin{abstract}
Background: Among the enterococci strains, Enterococcus faecalis is considered as one of the important nosocomial pathogens affecting immunocompromised patients. In this study, the immunogenicity of PpiC, GelE, and VS87_01105 proteins against enterococcal infection was investigated in a mice model. Methods: The genes encoding these proteins were cloned into PET21a expression vector, and the recombinant proteins were produced. Mice and rabbits were immunized with the purified recombinant proteins, and subsequently, mice were challenged with E. faecalis for the evaluation of their survival and bacterial clearances. The antibody responses to recombinant proteins were determined by ELISA assay, and opsonophagocytic activities of the antibodies were also measured. Passive immunization was performed using purified antibodies. Mice were challenged, and their survival and bacterial clearance were determined. Results: Immunized mice with PpiC, GelE, and VS87_01105 recombinant proteins showed $80 \%, 70 \%$, and $40 \%$ survival rate, respectively. The survival rates among passively immunized mice that received $500 \mu \mathrm{g}$ of IgG fraction in $100 \mu \mathrm{l}$ PBS buffer of each of anti-PpiC, anti-GelE, and anti-VS87_01105 were 60\%, 50\%, and 20\%, respectively. The rates of opsonization with anti-PpiC, anti-GelE, and anti-VS87_01105 antibodies at 1/10 dilution were $77 \%, 64 \%$, and $23 \%$, respectively. Conclusion: Based on our findings, PpiC, and GelE proteins can protect the mice against E. faecalis ATCC 29212 and effectively induce a protective antibody response. Thus, these proteins could be used as an additional therapeutic tool against enterococcal infections. Further studies to determine the role of PpiC in ligand binding and demonstration of epitope mapping may establish a credible target for vaccination. DOI: 10.29252/ibj.23.5.344
\end{abstract}

Keywords: Enterococcus, Immunogenicity, Molecular cloning

Corresponding Author: Mohammad Reza Pourmand

Department of Pathobiology, School of Public Health and Urology Research Center, Tehran University of Medical Sciences, Tehran, Iran;

Mobile: (+98-912) 5168520; E-mail: mpourmand@tums.ac.ir 


\section{INTRODUCTION}

$\mathrm{E}$ nterococci are considered as a part of the normal flora in humans and animals ${ }^{[1]}$. However, these bacteria may act as opportunistic pathogens in immunocompromised and hospitalized individuals ${ }^{[2]}$. In this condition, enterococci can cause a variety of clinical syndromes, including infective endocarditis ${ }^{[3]}$, bacteremia and sepsis $^{[4]}$, surgical site infections ${ }^{[5]}$, neonatal meningitis ${ }^{[6]}$, cholecystitis $^{[7]}$, peritonitis ${ }^{[8]}$, central nervous system infections ${ }^{[9]}$, wound infections ${ }^{[10]}$, and urinary tract infections ${ }^{[11]}$. Enterococcus faecalis, the most common isolate of nosocomial infections, accounts for more than $80-90 \%$ of human enterococcal infections ${ }^{[12]}$.

The emergence of resistant enterococci appears to have accelerated in the past decade. E. faecalis has been shown to have potential to acquire resistance against some antimicrobial agents, especially vancomycin ${ }^{[13]}$. Meanwhile, resistance to the last-resort antibiotics, such as daptomycin and linezolid, is also emerging among enterococcus species ${ }^{[14,15]}$. This trend has raised a serious concern about the treatment of infected individuals. Moreover, horizontal gene transfer has been reported to play an essential role in the spread of resistant enterococci to other susceptible species ${ }^{[16]}$. Consequently, treatment of these infections is becoming increasingly challenging and might lead to increases in patient morbidity, mortality, and healthcare costs ${ }^{[17]}$.

There is an urgent need to explore alternative strategies against enterococcal infections. Different surface antigens have been identified in E. faecalis that may be promising candidates for the development of vaccine against enterococcal infections. Only a few of these antigens have been tested in appropriate animal models $^{[2]}$. In E. faecalis, PpiC homologue has been identified as a potential virulence factor. The protein confers resistance to high $\mathrm{NaCl}$ concentrations and ampicillin, and it is also involved in the folding and trafficking of extracellular proteins, especially penicillin binding proteins $(\mathrm{PBPs})^{[18]}$. Gelatinase, encoded by gelE gene, is an extracellular metalloendopeptidase that hydrolyzes collagen, gelatin, and small peptides. This protein is important for enterococcal virulence ${ }^{[19]}$. VS87_01105 is also a cell surface protein in E. faecalis that is being investigated in this study.

To the best of our knowledge, no appropriate prophylactic studies on these enterococcal proteins have been reported so $\mathrm{far}^{[18]}$. With the goal of introducing a new vaccine, the recombinant PpiC, GelE, and VS87_01105 were produced in E. coli host, and their immunogenic potentials were considered in a mouse model.

\section{MATERIALS AND METHODS}

\section{Expression and purification of recombinant proteins}

DNA of E. faecalis ATCC 29212 was extracted using a DNA extraction kit (Qiagen, Germany) according to the manufacturer's instructions. The extracted DNA was stored at $-20{ }^{\circ} \mathrm{C}$ until further analysis. Amplification of ppiC, gelE, and VS87_01105 genes was carried out using specific primers, shown in Table 1.

The amplified DNA fragments and pET21a expression vector (Novagen, USA) were digested with appropriate restriction enzymes (Fermentas, Germany). Ligations were carried out separately using T4 DNA ligase (Takara Shuzo Co., Japan) at $4{ }^{\circ} \mathrm{C}$ for 16 h. The ligation mixtures were transformed into $E$. coli DH5 $\alpha$ competent cells by a standard $\mathrm{CaCl}_{2 /}$ heat shock

transformation method. Bacterial colonies resistant to ampicillin were selected and confirmed by colony PCR using T7 primers. Recombinant vectors were extracted using the QIAprep spin miniprep kit (Qiagen, USA). Finally, the recombinant plasmids were confirmed by agarose gel electrophoresis and DNA sequence

Table 1. Primers used in the study.

\begin{tabular}{|c|c|c|c|}
\hline Primers & Oligonucleotides $\left(5^{\prime} \rightarrow 3^{\prime}\right)^{\mathrm{a}}$ & $\begin{array}{c}\text { Restriction } \\
\text { enzyme }\end{array}$ & $\begin{array}{c}\text { Amplicon } \\
\text { size (bp) }\end{array}$ \\
\hline ppiC-F & GCGCGCCATATGACGACCGCAACGAGTGATTC & NdeI & \multirow{2}{*}{750} \\
\hline ppiC - R & 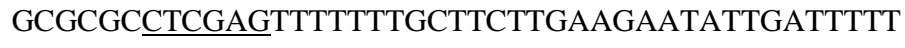 & XhoI & \\
\hline gelE-F & GCGCGCCATATGGCAGAAGAACAAGTTTATTCAGAAA & NdeI & \multirow{2}{*}{1530} \\
\hline gelE $-\mathrm{R}$ & GCGCGCCTCGAGTTCATTGACCAGAACAGATTCAC & XhoI & \\
\hline VS87_01105-F & GCGCGCCATATGGCAGAAGAACAAGTTTATTCAGAAA & NdeI & \multirow{2}{*}{1887} \\
\hline VS87_01105-R & GCGCGCCTCGAGTTCATTGACCAGAACAGATTCAC & XhoI & \\
\hline
\end{tabular}

${ }^{a}$ Artificial restriction sites are underlined. 
analysis. Expression of recombinant proteins in E. coli BL21 (DE3) and purification of the recombinant proteins were performed as described before ${ }^{[20]}$.

\section{Western-blot analysis}

Western-blot analysis was performed to confirm the successful protein expression using His-tag monoclonal antibody conjugated to horseradish peroxidase (HRP; Thermo Fisher Scientific, Lithuania). The recombinant proteins were separated on a $12.5 \%$ polyacrylamide SDS gel. The protein bands were then transferred onto PVDF membrane using a semi-dry blotting system (Bio-Rad, Hercules, CA, USA) at $4{ }^{\circ} \mathrm{C}$ for $90 \mathrm{~min}$. Membranes were blocked by incubation in PBS containing 3\% skimmed milk and $0.05 \%$ Tween 20 at $4{ }^{\circ} \mathrm{C}$ overnight. After blocking, membranes were washed three times with PBS containing $0.05 \%$ Tween 20 and then incubated with a 1:1000 dilution of anti-His Tag HRP-conjugated monoclonal antibody at $25{ }^{\circ} \mathrm{C}$ for $1 \mathrm{~h}$. Finally, the membranes were washed three times with PBS 1× containing $0.05 \%$ Tween 20 and exposed to 3,3diaminobenzidine solution (Sigma-Aldrich, USA) until the appearance of bands.

\section{Mice immunizations}

This study was conducted using 6-8-week-old BALB/c mice (Ethical number: IR.TUMS.SPH.REC. 1396.2067). The mice were obtained from Pasteur Institute of Iran (Karaj, Iran) and kept in cages in an animal house facility. Experiments were performed in accordance with animal protocols approved by the Institutional Animal Care and Use Committee of Tehran University of Medical Sciences. Complete Freund's adjuvant was used for initial injections and incomplete Freund's adjuvant for subsequent boosts. Mice were divided into four groups with 16 mice in each group, namely PpiC + adjuvant, GelE + adjuvant, VS87_01105+ adjuvant, and PBS. The three first groups were immunized with adjuvant $+30 \mu \mathrm{g}$ of the corresponding protein. Also, negative control group of mice was injected with PBS buffer. Entirely, all mice were subcutaneously immunized every 14 days to a total of three doses. To check antibody titers, before each immunization, blood samples were obtained from mice by tail bleeding.

\section{Rabbit immunizations}

White New Zealand male rabbits were purchased from Pasteur Institute of Iran. They weighed between $2-3 \mathrm{~kg}$. All the rabbits were immunized by multi-point injection in the back with adjuvant along with $50 \mu \mathrm{g}$ of recombinant proteins, 3 times at 14-day intervals. For ELISA identification, blood samples were taken from the marginal vein of the ear after seven days of each immunization. Seven days after the final boost, all immunized rabbits were exsanguinated by heart puncture, and the serum was separated from blood cells by storing at $37{ }^{\circ} \mathrm{C}$ for $1 \mathrm{hr}$ and centrifugation at $3000 \times \mathrm{g}$ at room temperature for $15 \mathrm{~min}$. Antibodies were purified using protein A affinity column chromatography (Bio-Rad Laboratories, Richmond, California, USA). The purified antibodies were dispensed into suitable containers and quantified with Bradford and stored at $-20{ }^{\circ} \mathrm{C}$.

\section{Enzyme-linked immunosorbent assay (ELISA)}

The ELISA assay was performed after last immunization to determine the specific total $\mathrm{IgG}$ antibody, $\mathrm{IgG}_{1}$, and $\mathrm{IgG}_{2} \mathrm{a}$ isotypes. The recombinant proteins $(10 \mu \mathrm{g} / \mathrm{ml}$ in PBS) were coated into 96-well polystyrene plates (Greiner Bio-One, Frickenhausen, Germany) by overnight incubation at $4{ }^{\circ} \mathrm{C}$. The wells were washed three times with PBS Tween 20 and then blocked with PBS containing 4\% skimmed milk at RT for $1 \mathrm{~h}$. The plates were then washed three times using PBS-T20, and diluted sera were added into the wells at the dilutions of $1: 100$ to $1: 12800$ and incubated at RT for $1 \mathrm{~h}$. Following rinses, peroxidase-conjugated goat anti-mouse IgG (Cyto Matin Gene, Isfahan, Iran; diluted 1:2000) was added and incubated at room temperature for one hour. Finally, tetra-methyl benzidine (TMB) substrate solution (Cyto Matin Gene) was added, and the reaction was stopped with adding $100 \mu \mathrm{l}$ of $2 \mathrm{~N} \mathrm{H}_{2} \mathrm{SO}_{4}$.

\section{Cytokine assay}

For cytokine ELISA assay, blood samples were obtained from mice by retro-orbital bleeding after $16 \mathrm{~h}$ of the last immunization. Sera were separated from blood cells by storing at $37{ }^{\circ} \mathrm{C}$ for one hour and centrifugation at $12000 \times \mathrm{g}$ at room temperature for $10 \mathrm{~min}$. The samples were analyzed for IL-4, IL-17, and IFN- $\gamma$ using a sandwich ELISA assay kit (Mabtech, Sweden) according to the manufacturer's recommendations.

\section{Opsonophagocytic assay}

The opsonophagocytic assay was performed according to the method of Ames et al. ${ }^{[21]}$. Briefly, bacterial suspensions were prepared at the concentration of $1 \times 10^{8} \mathrm{CFU} / \mathrm{mL}$ in $1 \%$ bovine serum albumin (BSA). A final concentration of $2 \times 10^{7} \mathrm{~mL}$ mouse macrophages in RPMI-1640 supplemented with $10 \%$ heat-inactivated fetal bovine serum was used. Besides, baby rabbit sera (Pasture Institute of Iran) were used as a complement source, and a dilution of pooled pre-challenge anti-r-PpiC, anti-r-GelE, and anti- 


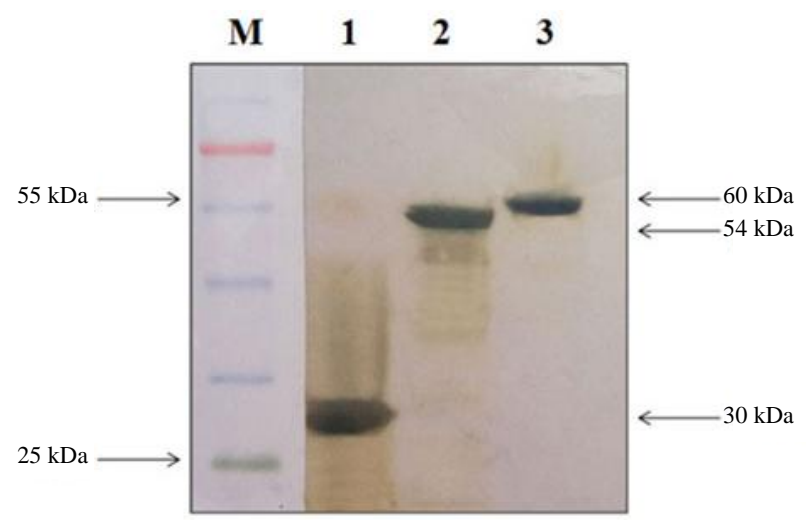

Fig. 1. Western-blot analysis of recombinant proteins. Lane 1, purified PpiC; lane 2, GelE (2); lane 3, VS87_0110; M, protein size marker

r-VS87_01105 from each group was used. Complement of antisera was inactivated by heating at $56{ }^{\circ} \mathrm{C}$ for $30 \mathrm{~min}$. For the opsonophagocytic assay, the bacteria were incubated with an equal 1:10 volume of heat-inactivated polyclonal $\mathrm{IgG}$ at $22{ }^{\circ} \mathrm{C}$ for $1 \mathrm{~h}$. To eliminate excessive antibodies, wells were washed twice with BSA $(1 \%[\mathrm{w} / \mathrm{v}])$. Then $100 \mu \mathrm{L}$ of mouse macrophage was mixed with $100 \mu \mathrm{L}$ of complement in a sterile 96-well plate (Greiner Bio-One) and incubated in a shaker at $37{ }^{\circ} \mathrm{C}$ for $90 \mathrm{~min}$. Finally, $25 \mu \mathrm{L}$ of the mixture was removed, diluted in saline and plated for bacterial enumeration. The opsonic activity of immune sera was compared to non-immune normal rabbit serum (NRS), statistically. This experiment was performed in triplicate for each quantity. The following formula was used for calculation of the percentage of killed bacteria.

Opsonophagocytosis $(\%)=(1-[\mathrm{CFU}$ of immune serum/CFU of pre-immune serum] $) \times 100$

\section{Experimental challenge}

A mouse bacteremia model was induced in the immunized mice and control groups with E. faecalis ATCC $29212\left(1 \times 10^{8} \mathrm{CFU}\right)$. Bacterial inoculum was injected via the tail vein (intra-venus [i.v.]). Twentyfour hours after challenge, the blood collection was performed from the tail vein of the mice and diluted serially into PBS and were then cultured on blood agar plates. This procedure was followed until seven days after infection. Moreover, mice were observed daily for mortality until 14 days after infection.

\section{Passive protection assays}

To explore the protectivity of rabbit $\mathrm{IgG}$ antibodies fraction against PpiC, VS87_01105, and GelE, 10 female $\mathrm{BALB} / \mathrm{c}$ mice were employed in each experimental group. The dose of $500 \mu \mathrm{g}$ of $\mathrm{IgG}$ fraction in $100 \mu \mathrm{l}$ of PBS buffer was used for each antibody. Then equal volumes of bacterial suspension (approximately $1 \times 10^{8} \mathrm{CFU}$ ) and $500 \mu \mathrm{g}$ of $\mathrm{IgG}$ antibody fraction were administered i.v. to 10 mice in each group. The CFU was counted out at 1, 2, 3, and 7 day(s) after bacterial challenge. The survival time of each mouse was monitored daily for 21 days.

\section{Statistical analyses}

The Kruskal-Wallis test was used for the statistical analysis of the data. The groups with significant differences were further analyzed by post hoc tests. The limit of statistical significance was a $p$ value of 0.05. All data analyses were performed using the Statistical Packages for Social Sciences (SPSS) version 22 .

\section{RESULTS}

\section{Purification and characterization of recombinant proteins}

All three recombinant proteins were expressed as inclusion body in E. coli expression host BL21 (DE3). The recombinant proteins were then purified by $\mathrm{Ni}$ NTA chromatography. All the recombinant proteins were of predicted size based on the migration on SDSPAGE gels (approximately $60 \mathrm{kDa}$ for GelE, $55 \mathrm{kDa}$ for VS87_01105, and $30 \mathrm{kDa}$ for PpiC). Western-blot analysis using anti-His monoclonal antibody revealed positive bands with the correct size of recombinant proteins (Figs. 1 and 2).

\section{Mice immune response to antigens}

Experimental sera collected from the mice after the last booster injection were assessed in all groups of

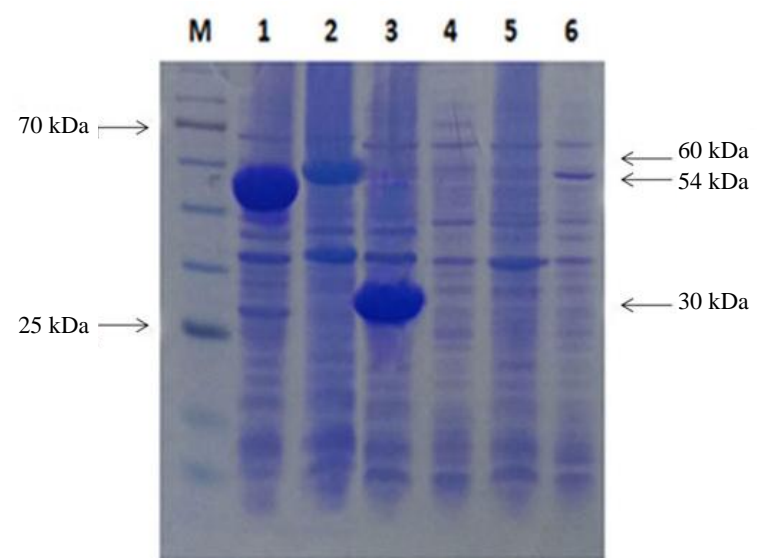

Fig. 2. Expression of PpiC, GelE, and VS87_01105 proteins in $12.5 \%$ gel agarose. Lanes 1, 2, and 3, GelE, VS87_01105, and PpiC proteins after induction, respectively; lanes 3, 4, and 5, GelE, VS87_01105, and PpiC proteins before induction, respectively. $\mathrm{M}$, protein size marker 


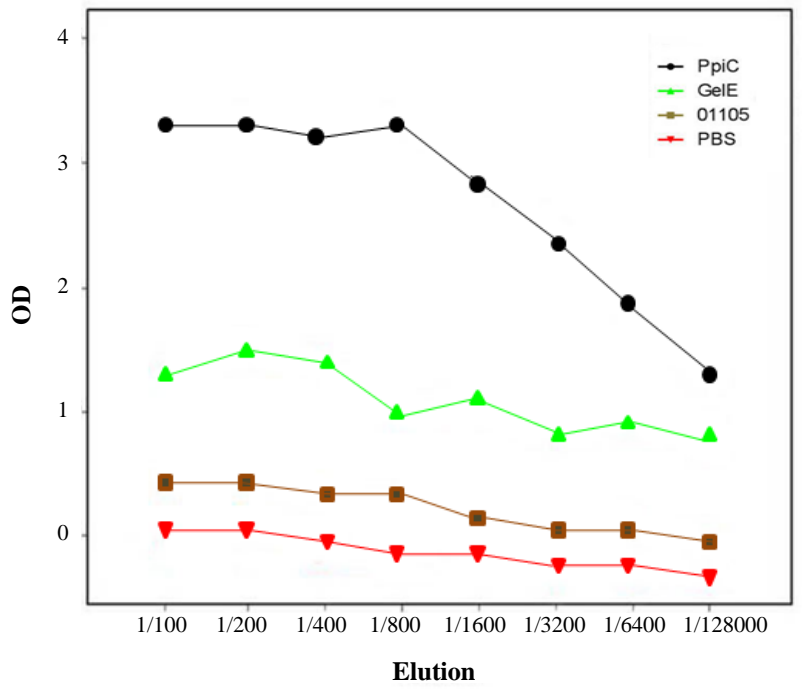

Fig. 3. Titration of specific antibodies in sera of immunized mice. Two weeks after the last immunization, serum samples of the mice were collected and pooled. The sera were serially diluted and were coated on recombinant proteins. Different groups of mice each consisting of 16 mice were administrated with either the recombinant antigens PpiC, GelE, and VS87_01105. Mice injected with PBS alone served as controls. The titers of specific antibodies were significantly high in mice $(p<0.05)$.

mice by indirect-ELISA. Data analysis showed that antibody titers PpiC and GelE recombinant proteins induced a significant antibody response in mice when compared with the PBS. VS87_01105 protein, however, did not induce any significant antibody response in the mice model $(p<0.05$; Fig. 3). The types of immune responses to PpiC, VS87_01105 and GelE antigens were further examined by measuring the levels of $\mathrm{IgG}_{1}$ and $\mathrm{IgG}_{2}$ a isotypes. The $\mathrm{IgG}_{1}$ levels were higher than $\operatorname{IgG}_{2}$ a levels in comparison to the control groups with statistical significance $(p<0.05$; Fig. 4).

\section{Survival rate and bacterial clearance in immunized mice}

To evaluate the protection elicited by immunization with recombinant PpiC, GelE, and VS87_01105 proteins, BALB/c mice were immunized with these proteins (formulated in adjuvant) and then challenged with E. faecalis ATCC 29212. The mice immunized with the recombinant PpiC showed a statistically significant improvement $(p<0.05)$ in survival rate after bacterial challenge (Fig. 5). The median survival times for mice immunized with the experimental antigens were significantly longer than the times used for mice that received PBS alone $(p<0.05)$. The highest survival rate was observed in mice that received PpiC antigen. Administration of VS87_01105 antigen only delayed the time to death but failed to protect mice from death. The clearance rates from enterococcal bacteremia following immunization with PpiC, GelE, and VS87_01105 recombinant proteins were within $96 \mathrm{~h}$ after the challenge, although mice immunized with PpiC have been cleared at $48 \mathrm{~h}$ after infection (Fig. 6). The comparison of the immunized groups with the control group showed a significant difference at $24 \mathrm{~h}(p<$ $0.05)$, but no significant differences were seen among the immunized groups $(p>0.05)$.
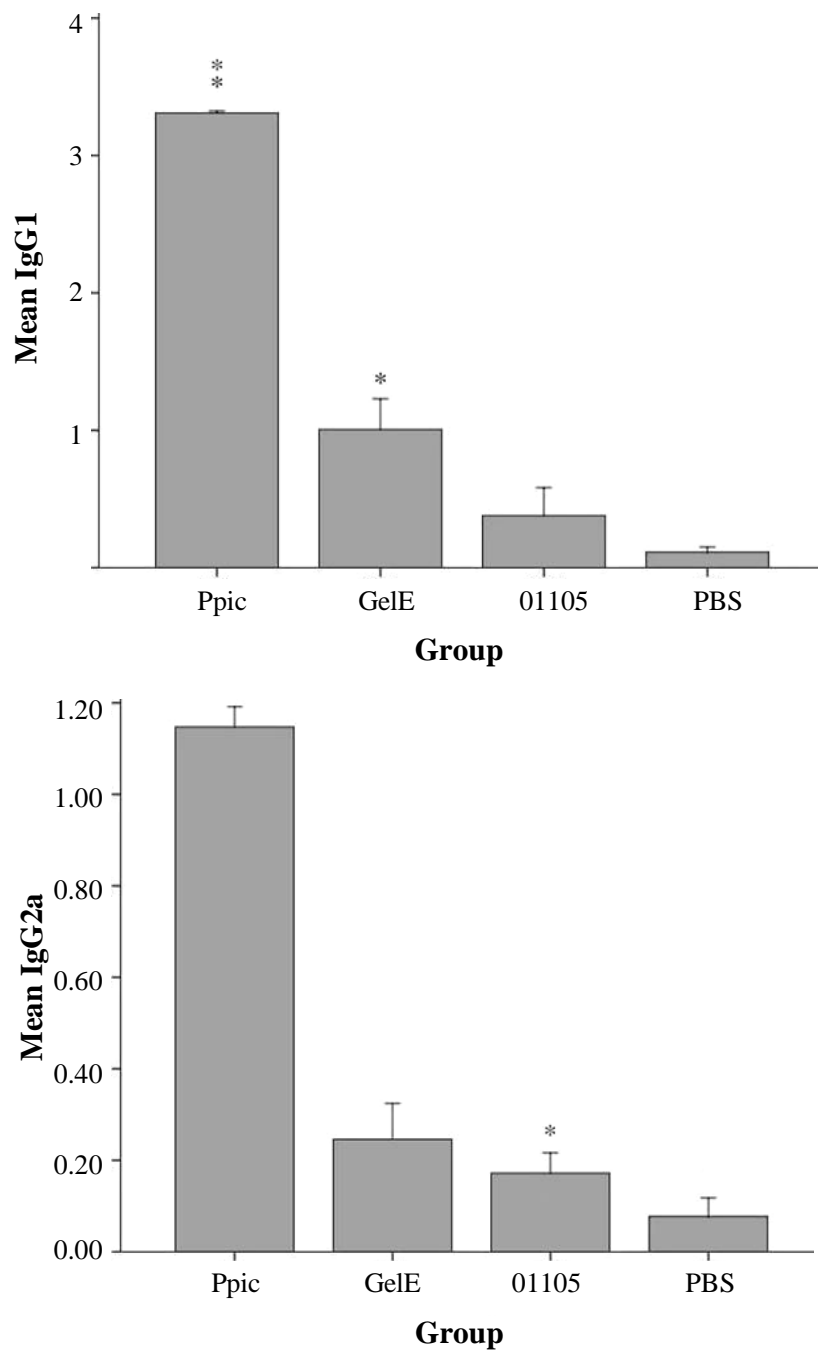

Fig. 4. Immunoglobulin isotyping assay in sera of immunized mice. Two weeks after the last immunization, serum samples of the mice were collected and pooled. The sera were serially diluted and were coated on recombinant proteins. Different groups of mice each consisting of 16 mice were administrated with either the recombinant antigens PpiC, GelE, and VS87_01105. Mice injected with PBS alone served as controls. The IgG1 levels were higher than $\mathrm{IgG}_{2}$ a levels in comparison to the control groups. The IgGs levels against PpiC were higher than other proteins. ${ }^{*} p<0.05$ and ${ }^{* *} p<0.01$ 


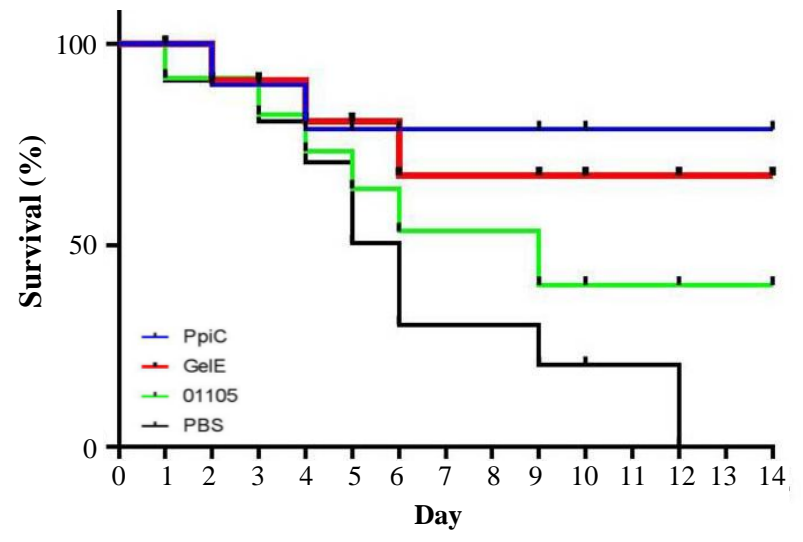

Fig. 5. Cumulative survival rates of mice challenged with the serotype E. faecalis ATCC 29212. The mice were intraperitoneally infected with $1 \times 10^{8} \mathrm{CFU}$ of E. faecalis. Survival rate of each group was checked for 14 days. There were statistically significant differences $(p<0.05)$ between the immunized mice and control groups.

\section{Cytokine assay}

A significant amount of IFN- $\gamma$ and IL-4 cytokines was produced in immunized mice, whereas the IL-17 cytokine level was very low (Fig. 7). There were not statistically significant differences $(p<0.05)$ between the groups. The level of IL-17 in the mice immunized with PpiC vaccine shows a significant difference $(p<$ $0.05)$ in comparison to other vaccinated and control groups.

\section{Opsonophagocytic activity}

The bioactivity of antibodies against recombinant proteins to promote phagocytosis of bacteria was evaluated by incubating E. faecalis with pooled and diluted anti-r-PpiC, anti-r-GelE, and anti-rVS87_01105 antibodies (pre-challenge serum) and mouse macrophages in the presence of rabbit complement. The anti-r-PpiC and anti-r-GelE sera induced a higher significant increase in the opsonophagocytic responses $(77 \%$ and $64 \%$, respectively) at a dilution of $1: 10$ than the VS87_01105 (23\%) sera groups against the E. faecalis ATCC 29212, two weeks after the last dose $(p<0.05)$. A statistically significant difference $(p<0.01)$ was observed in respiratory burst among the anti-r-PpiC and anti-r-GelE sera and NRS $(<1 \%)$, as shown in Figure 8 . There was no significant difference between anti- VS87_01105 and NRS $(p>0.05)$.

\section{Passive protection and enterococcal challenge}

To examine the protectivity of rabbit-specific IgG antibodies against enterococcal infection, mice were passively immunized with rabbit hyper-immune $\operatorname{IgG}$ fraction before bacterial challenge. Mice that received hyper-immune sera lived significantly longer than those received sera from non-immunized rabbits $(p<$ 0.05 ). The survival rates of the mice groups that received anti-PpiC, anti-GelE, and anti-VS87_01105 antibodies were $75 \%, 50 \%$ and $25 \%$, respectively (Figs. 9 and 10).

\section{DISCUSSION}

E. faecalis is considered as an important nosocomial pathogen in individuals whose immune systems are compromised $^{[22]}$. Enterococcal infections result from the capacity of the bacteria to adhere, colonize and invade onto host tissues and to produce extracellular enzymes and toxins as well as various virulence factors that enhance the severity of the infection ${ }^{[23]}$. Enterococcus is a problematic pathogen that is becoming increasingly hard to treat even with available antibiotics. The identification of protective antigens is needed to facilitate vaccine-based prophylactic approaches. Previous studies have demonstrated that surface-exposed proteins, closely related to peptidoglycan, can be targeted as potential candidates for vaccine production against enterococcal pathogens ${ }^{[18,24]}$.

E. faecalis has many surface proteins on its cell wall that play major physiological and structural roles. By in silico approaches, many surface-exposed proteins have been characterized to be promising vaccine candidates in different species of bacteria ${ }^{[25-27]}$. In this study, the immunogenicity and protectivity of PpiC, GelE, and VS87_01105 recombinant proteins on E. faecalis were investigated.

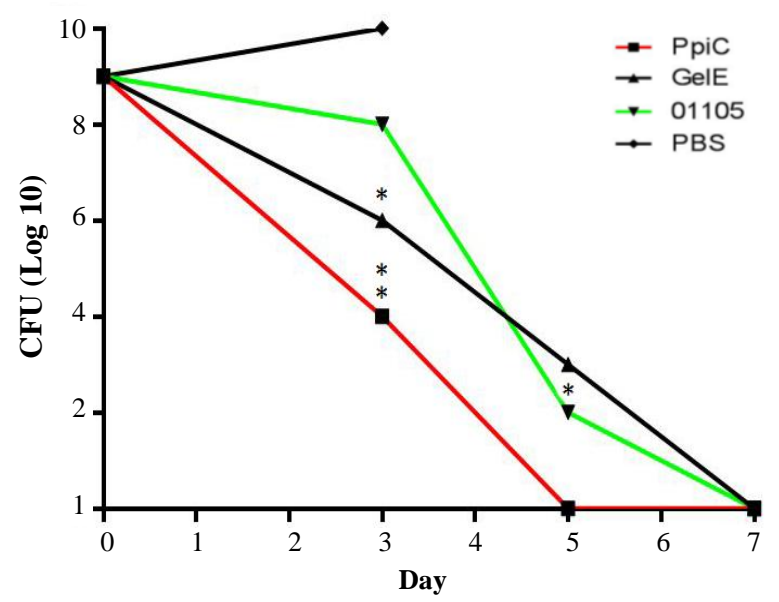

Fig. 6. Clearance rate of mice from enterococcal bacteremia. The mice were intraperitoneally infected with $1 \times 10^{8} \mathrm{CFU}$ of $E$. faecalis ATCC 29212. The CFUs in the blood were determined 3 , 5, and 7 days after challenge. There were statistically significant differences $(p<0.05)$ between the immunized mice and control groups. ${ }^{*} p<0.05$ and ${ }^{* *} p<0.01$ 

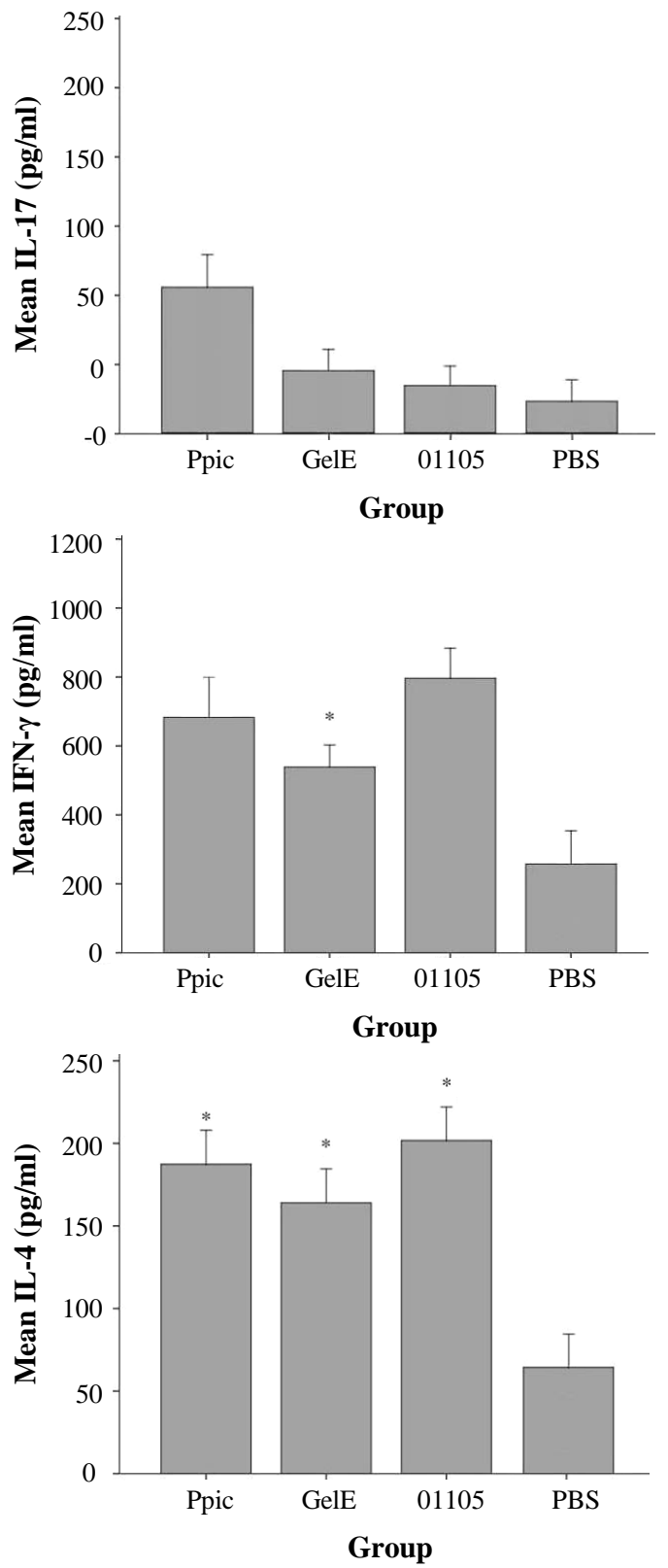

Fig. 7. Levels of IL-4, IL-17, and IFN- $\gamma$ cytokines $(\mathrm{pg} / \mathrm{ml})$ in mice groups. Control groups were not stimulated by antigens. The level of IL-17 was very low compared to the two other cytokines and did not show statistical differences with mice group that received PBS $(p>0.05)$. There were not statistically significant differences $(p>0.05)$ between the groups $\left({ }^{*} p<0.05\right)$.

Our results showed that the PpiC protein elicited protective antibodies in mice challenged with $E$. faecalis ATCC 29212. A similar study has demonstrated that cell surface proteins, including PipC could produce specific, opsonic and protective antibodies against enterococcal infections and thus can be considered as vaccine candidates ${ }^{[18]}$. Our results demonstrated immunogenic capacities of PpiC, GelE, and VS87_01105 recombinant proteins. Passive immunization with the antibodies raised against different proteins promoted the clearance of $E$. faecalis ATCC 29212 in the immunized mice in comparison with the normal mice sera. These results are comparable to the protection achieved by antibodies raised against the previously reported antigens, such as SagA and $\mathrm{LTA}^{[28,29]}$. The high clearance activity of PpiC in comparison to the two other proteins may be associated with the high immunogenicity and possible surface-exposing features in this protein, although this area requires further investigation. Similarly, the high survival rate of mice immunized with $\mathrm{PpiC}$ protein has formerly been reported ${ }^{[18]}$. In this study, recombinant proteins and PBS were emulsified with Freund's complete adjuvant (first vaccination) and Freund's incomplete adjuvant (boosts), which generated high titers of anti-PpiC and anti-GelE. This result is comparable to other study that used this $\operatorname{adjuvant}^{[30]}$.

In our study, the plasma level of IFN- $\gamma$ produced in mice immunized with PpiC, GelE, and VS87_01105 recombinant proteins was higher than that of IL-4 and IL-17. The high level production of this cytokine in response to recombinant proteins might be due to the stimulation of Th-1 lymphocytes. A number of studies have reported that certain cytokines and other components of innate immune systems are essential for bacterial clearance in animal models ${ }^{[31,32]}$. However, the plasma level of IFN- $\gamma$ raised only within six hour post enterococcal challenge ${ }^{[33]}$. Suppression of innate immune system by $E$. faecalis has been reported to be associated with the blockade of NF-KB signaling by reducing microbial clearance in the model mouse $^{[24]}$.

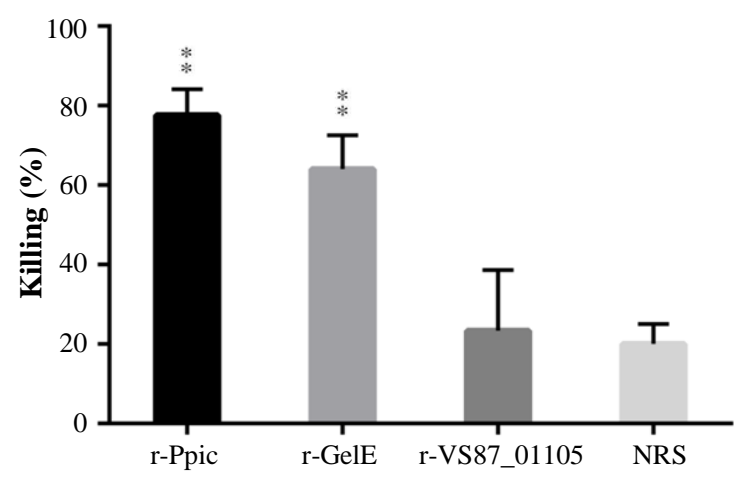

Fig. 8. The opsonic activity of PpiC, GelE, and VS87_01105 proteins. (A) At a dilution of 1:10, the anti-r-PpiC and antir-GelE sera induced a higher significant increase in the opsonophagocytic responses than the VS87_01105 sera groups against the E. faecalis ATCC 29212, two weeks after the last dose $(p<0.05)$. A statistically significant difference was observed among the anti r-PpiC, anti- GelE sera, and NRS. There is no significant difference between anti-VS87_01105 and NRS $(p>0.05) .{ }^{* *} p<0.01$ 


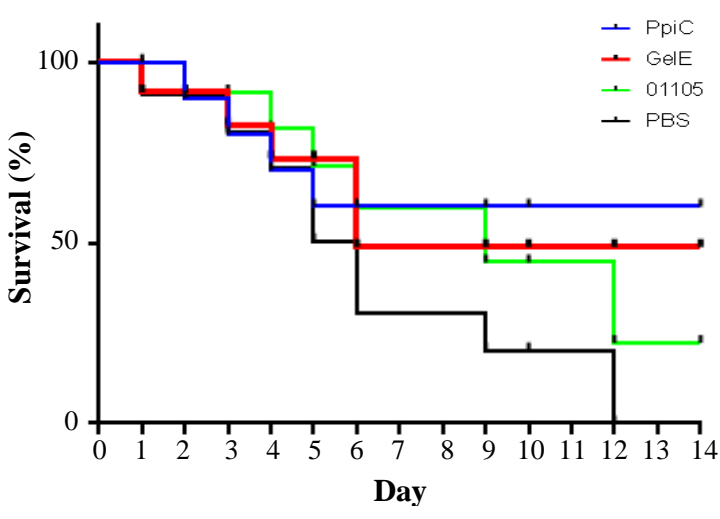

Fig. 9. The Kaplan-Meier curve (cumulative survival) of $\mathrm{BALB} / \mathrm{c}$ mice challenged with $E$. faecalis. The cumulative survival rate was plotted against time. BALB/c mice immunized with the recombinant PpiC showed a statistically significant improvement $(p<0.05)$ in survival rate after bacterial challenge with E. faecalis ATCC 29212 compared to other mice groups. antibodies in bacterial opsonization, need to be further tested $^{[29,35]}$.

In a previous study that conducted by Leendertse et $a l .{ }^{[34]}$ certain enterococcal protein has elicited specific opsonic antibodies that could mediate opsophogocytic killing in immunized animal models. In our study, we demonstrated that PpiC and GelE proteins induced a significant increase in the opsonophagocytic responses (77\% and $64 \%$, respectively) against the E. faecalis ATCC 29212. The opsonophagocytic activity of the sera was found to be dose-dependent, with maximum

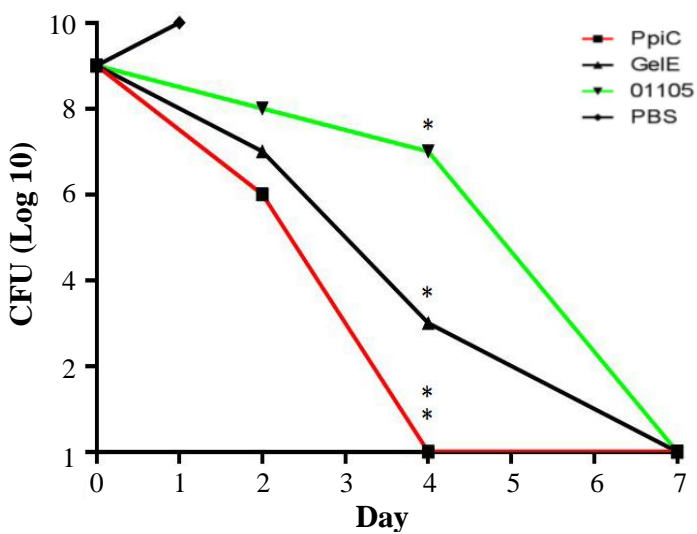

Fig. 10. Enterococcal clearance of mice receiving polyclonal antibodies. The clearance rates from enterococcal bacteremia also indicated that immunization with PpiC, GelE, and VS87_01105 recombinant proteins resulted in bacterial clearance within seven days after the challenge, although mice immunized with PpiC were cleared at $96 \mathrm{~h}$ after infection. The comparison of immunized groups with each other and control groups showed a significant difference at $96 \mathrm{~h}$ with control groups $(p<0.05)$, while no significant differences were seen among the immunized groups $(p>0.05) .{ }^{*} p<0.05$ and ${ }^{* *} p<0.01$ activity at 1:10 dilution. A similar pattern of activity has also been reported in a previous study ${ }^{[34]}$. Although the recombinant proteins induce high levels of antibodies, antibodies against VS87_01105 protein have been shown to have a low level of opsonic activity. We suppose that this protein may not be adequately exposed to the antibodies. This insufficient exposure may be due to difference in opsonic capacity of antibody subtypes. Other antibody subtypes such as IgM antibodies, which are more effective than IgG The protection offered by passive immunization indicates that the defense against entrococcal infections obtained from a balanced Th1 and Th2 stimulatory response owing to significant plasma level of INF- $\gamma$ and IL-4 in the immunized models. The effectiveness of passive protection against enterococcal infections using sera from mice immunized with other surface proteins has also been reported ${ }^{[18]}$. Such findings reveal that these surface-related proteins can be promising vaccine candidates for enterococcal infections.

Results of the present research clearly demonstrate that the PpiC recombinant protein efficiently stimulate cell-mediated immunity and results in significant opsonophagocytotic activity against $E$. faecalis ATCC 29212 infection. Our results predict that this enterococcal polysaccharide protein has the potential for broad protective coverage against $E$. faecalis infection. Both PpiC and GelE proteins can be used as candidates for vaccine development, especially in combined form for better efficacy. Further studies to determine the role of PpiC in ligand binding and demonstrate the epitope mapping may qualify the protein as a credible target for vaccination.

\section{ACKNOWLEDGEMENTS}

This work was supported by a grant from Tehran University of Medical Sciences (No. 32761, 17 may 2017). We thank all the staffs of the Pathobiology Department at TUMS for their technical and intellectual assistance.

CONFLICT OF INTEREST. None declared.

\section{REFERENCES}

1. Arshadi M, Mahmoudi M, Motahar MS, Soltani S, Pourmand MR. Virulence determinants and antimicrobial resistance patterns of vancomycinresistant Enterococcus faecium isolated from different sources in Southwest Iran. Iranian journal of public health 2018; 47(2): 264-272.

2. Sava IG, Heikens E, Huebner J. Pathogenesis and 
immunity in enterococcal infections. Clinical microbiology and infection 2010; 16(6): 533-540.

3. McDonald JR, Olaison L, Anderson D J, Hoen B, Miro JM, Eykyn S, Abrutyn E, Fowler VG Jr, Habib G, Selton-Suty C, Pappas PA, Cabell CH, Corey GR, Marco F, Sexton DJ. Enterococcal endocarditis: 107 cases from the international collaboration on endocarditis merged database. The American journal of medicine 2005 ; 118(7): 759-766.

4. Suppli M, Aabenhus R, Harboe ZB, Andersen LP, Tvede M, Jensen JU. Mortality in enterococcal bloodstream infections increases with inappropriate antimicrobial therapy. Clinical microbiology and infection 2011; 17(7): 1078-1083.

5. Giacometti A, Cirioni O, Schimizzi AM, Del Prete MS, Barchiesi F, D'Errico MM, Petrelli E, Scalise G. Epidemiology and microbiology of surgical wound infections. Journal of clinical microbiology 2000; 38(2): 918-922.

6. Bretón JR, Peset V, Morcillo F, Cano J, Sarrión A, Pérez-Belles C, Gobernado M. Neonatal meningitis due to Enterococcus spp.: presentation of four cases. Enfermedades infecciosas microbiologia clinica 2002; 20(9): 443-447.

7. Khardori N, Wong E, Carrasco CH, Wallace S, Patt Y, Bodey GP. Infections associated with biliary drainage procedures in patients with cancer. Reviews of infectious diseases 1991; 13(4): 587-591.

8. Perez-Fontán M, Rodriguez-Carmona A, RodríguezMayo M. Enterococcal peritonitis in peritoneal dialysis patients: last name matters. Peritoneal dialysis international 2011; 31(5): 513-517.

9. Patel T, Lewis ME, Niesley ML, Chowdhury M. Postneurosurgical central nervous system infection due to Enterococcus faecalis successfully treated with intraventricular vancomycin. Infectious diseases in clinical pratice (Baltim Md) 2016; 24(3): 174-176.

10. Sabzi S, Mashhadi R, Pourmand MR. Fibrinogen and mucin binding activity of EF0737, a novel protein of Enterococcus faecalis. Iranian journal of microbiology 2017; 9(6): 324-330.

11. Fallah F, Yousefi M, Pourmand MR, Hashemi A, Nazari Alam A, Afshar D. Phenotypic and genotypic study of biofilm formation in Enterococci isolated from urinary tract infections. Microbial pathogenesis 2017; 108: 8590.

12. Fernandes SC, Dhanashree B. Drug resistance and virulence determinants in clinical isolates of Enterococcus species. Indian journal of medical research 2013; 137(5): 981-985.

13. Arshadi M, Douraghi M, Shokoohizadeh L, Moosavian SM, Pourmand MR. High prevalence of diverse vancomycin resistance Enterococcus faecium isolates in clinical and environmental sources in ICU wards in southwest of Iran. Microbial pathogenesis 2017; 111: 212-217.

14. Munoz-Price LS, Lolans K, Quinn JP. Emergence of resistance to daptomycin during treatment of vancomycin-resistant Enterococcus faecalis infection. Clinical infectious diseases 2005; 41(4): 565-566.
15. Giridhara Upadhyaya PM, Ravikumar KL, Umapathy BL. Review of virulence factors of enterococcus: an emerging nosocomial pathogen. Indian journal of medical microbiology 2009; 27(4): 301-305.

16. Jain H, Mulay S, Mullany P. Persistence of endodontic infection and Enterococcus faecalis: Role of horizontal gene transfer. Gene reports 2016; 5: 112-116.

17. Miller WR, Murray BE, Rice LB, Arias CA. Vancomycin-resistant enterococci: therapeutic challenges in the 21st century. Infectious disease of North America 2016; 30(2): 415-439.

18. Romero-Saavedra F, Laverde D, Wobser D, Michaux C, Budin-Verneuil A, Bernay B, Benachour A, Hartke A, Huebner J. Identification of peptidoglycan-associated proteins as vaccine candidates for enterococcal infections. PLoS one 2014; 9(11): e111880.

19. Stinemetz EK, Gao P, Pinkston KL, Montealegre MC, Murray BE, Harvey BR. Processing of the major autolysin of E. faecalis, AtlA, by the zincmetalloprotease, GelE, impacts AtlA septal localization and cell separation. PLoS one 2017; 12(10): e0186706.

20. Majelan PA, Mahdavi M, Yazdi MH, Salimi E, Pourmand MR. Recombinant Staphylococcal Antigen-F (r-ScaF), a novel vaccine candidate against methicillin resistant Staphylococcus aureus infection: Potency and efficacy studies. Microbial pathogenesis 2018; 127: 159-165.

21. Ames P, DesJardins D, Pier GB. Opsonophagocytic killing activity of rabbit antibody to Pseudomonas aeruginosa mucoid exopolysaccharide. Infection and immunity 1985; 49(2): 281-285.

22. Holmberg A, Rasmussen M. Mature biofilms of Enterococcus faecalis and Enterococcus faecium are highly resistant to antibiotics. Diagnostic microbiology and infectious disease 2016; 84(1): 19-21.

23. Strateva T, Atanasova D, Savov E, Petrova G, Mitov I. Incidence of virulence determinants in clinical Enterococcus faecalis and Enterococcus faecium isolates collected in Bulgaria. The Brazilian journal of infectious diseases 2016; 20(2): 127-133.

24. Tien BYQ, Goh HMS, Chong KKL, Bhaduri-Tagore S, Holec S, Dress R, Ginhoux F, Ingersoll MA, Williams $\mathrm{RBH}$, Kline KA. Enterococcus faecalis promotes innate immune suppression and polymicrobial catheterassociated urinary tract infection. Infection and immunity 2017; 85(12): pii: e00378-17.

25. Janulczyk R, Rasmussen M. Improved pattern for genome-based screening identifies novel cell wallattached proteins in gram-positive bacteria. Infection and immunity 2001; 69(6): 4019-4026.

26. Rodriguez-Ortega MJ, Norais N, Bensi G, Liberatori S, Capo S, Mora M,Scarselli M, Doro F, Ferrari G, Garaguso I, Maggi T, Neumann A, Covre A, Telford JL, Grandi G. Characterization and identification of vaccine candidate proteins through analysis of the group A Streptococcus surface proteome. Nature biotechnology 2006; 24(2): 191-197.

27. Hempel K, Herbst FA, Moche M, Hecker M, Becher, D. Quantitative proteomic view on secreted, cell surfaceassociated, and cytoplasmic proteins of the methicillin- 
resistant human pathogen Staphylococcus aureus under iron-limited conditions. Journal of proteome research 2011; 10(4): 1657-1666.

28. Kropec A, Sava IG, Vonend C, Sakinc T, Grohmann E. Huebner J. Identification of SagA as a novel vaccine target for the prevention of Enterococcus faecium infections. Microbiology 2011; 157(Pt 12): 3429-3434.

29. Kodali S, Vinogradov E, Lin F, Khoury N, Hao L, Pavliak V, Jones H, Laverde D, Huebner J, Jansen KU, Anderson AS, Donald RG. A vaccine approach for the prevention of infections by multidrug resistant Enterococcus faecium. Journal of biological chemistry 2015; 290(32): 19512-19526.

30. Flores-Mireles AL, Pinkner JS, Caparon MG, Hultgren SJ. EbpA vaccine antibodies block binding of Enterococcus faecalis to fibrinogen to prevent catheterassociated bladder infection in mice. Science translational medicine 2014; 6(254): 254ra127.

31. Park $\mathrm{S}, \mathrm{Nahm} \mathrm{MH}$. Older adults have a low capacity to opsonize pneumococci due to low IgM antibody response to pneumococcal vaccinations. Infection and immunity 2011; 79(1): 314-320.

32. Pollack M, Koles NL, Preston MJ, Brown BJ, Pier GB. Functional properties of isotype-switched immunoglobulin $\mathrm{M}(\mathrm{IgM})$ and $\operatorname{IgG}$ monoclonal antibodies to Pseudomonas aeruginosa lipopolysaccharide. Infection and immunity 1995; 63(11): 4481-4488

33. Leendertse M, Willems RJ, Giebelen IA, Roelofs JJ, van Rooijen N, Bonten MJ, van der Poll T. Peritoneal macrophages are important for the early containment of Enterococcus faecium peritonitis in mice. Innate immunity 2009; 15(1): 3-12.

34. Leendertse M, Willems RJ, Flierman R, de Vos AF, Bonten MJ, van der Poll T. The complement system facilitates clearance of Enterococcus faecium during murine peritonitis. Journal of infectious diseases 2010; 201(4): 544-552.

35. Leendertse M, Willems RJ, Giebelen IA, Roelofs JJ, Bonten MJ, van der Poll T. Neutrophils are essential for rapid clearance of Enterococcus faecium in mice. Infection and immunity 2009; 77(1): 485-491. 